\title{
Computation of Local Fractal Dimension Values of the Human Cerebral Cortex
}

\author{
Richard D. King \\ Center for Alzheimer's Care, Imaging and Research, Department of Neurology, University of Utah, Salt Lake \\ City, USA \\ Email: richard.king@hsc.utah.edu
}

Received 25 April 2014; revised 26 May 2014; accepted 4 June 2014

Copyright (C) 2014 by author and Scientific Research Publishing Inc.

This work is licensed under the Creative Commons Attribution International License (CC BY).

http://creativecommons.org/licenses/by/4.0/

(c) () Open Access

\begin{abstract}
Objectives: The purpose of this paper is to describe a technique for computing the local fractal dimension of the human cerebral cortex as extracted from high-resolution magnetic resonance imaging scans. Methods: 3D models of the human cerebral cortex were extracted from high resolution magnetic resonance images of 10 healthy adult volunteers using FreeSurfer. The local fractal dimension of the cortex was computed using a custom-written cube-counting algorithm. The effect of constraining the maximum region size on the measured value of local fractal dimension was examined. A proof of principle was demonstrated by comparing an individual with Alzheimer's disease to a healthy individual. Results: Local values of cortical fractal dimension can be obtained by constraining the size of the region over which the cube counting is performed. Cubic regions of intermediate size $(30 \times 30 \times 30 \mathrm{~mm})$ yielded a profile that demonstrated greater regional variability compared to smaller $(15 \times 15 \times 15 \mathrm{~mm})$ or larger $(60 \times 60 \times 60 \mathrm{~mm})$ region sizes. Conclusions: Local fractal dimension of the cerebral cortex is a novel measure that may yield additional, quantitative insight into the clinical meaning of cortical shape changes.
\end{abstract}

\section{Keywords}

Fractal, Dimension, Cortex, Imaging Biomarkers, Local

\section{Introduction}

Fractal dimension analysis was first made popular by a series of works by Benoit Mandelbrot in the late 1970s and early 1980s [1] [2]. Mathematically created fractal objects, such as Cantor dust or the Sierpinski gasket, exhibit a property called "self-similarity", which means that magnification of smaller scale features exactly duplicates a larger scale structure. In nature, many objects such as plants (ferns, cauliflower), clouds, mountains, and rivers also exhibit a property of statistical self-similarity. While mathematical objects have an infinite range to 
their self-similarity, biological objects only exhibit this property over a limited spatial range. The analytic techniques using this principle can model very complicated structures using relatively simple computational algorithms.

Fractal analysis has been applied to many fields of natural science including botany, geology, and medicine, to name a few. One particular application that has been advanced in the past decade is the use of fractal dimension as a measure of the complexity of the human cerebral cortex. Studies using anatomical data from either tissue specimens or magnetic resonance images have demonstrated that the human cerebral cortex exhibits fractal properties which arise secondary to cerebral folding [3]-[8]. Fractal analysis has been used to study several neurological conditions, including schizophrenia [9]-[12], multi-systems atrophy [13], epilepsy [14], stroke [15], cortical development [16] [17], and Alzheimer's disease [18] [19].

When applied to the cerebral cortex, the information obtained by the analysis of fractal dimension is related to, but different from, information obtained using standard volumetric approaches (such as measures of brain volume, surface area, cortical thickness, or gyrification index). Fractal analysis measures integrate information over a range of spatial scales (two orders of magnitude from $0.5 \mathrm{~mm}$ to $\sim 30 \mathrm{~mm}$ ). This unique approach to shaping analysis can integrate several aspects of structural change associated with disease (i.e. both subtle changes in cortical thickness associated with synaptic and neuronal loss as well as larger scale changes in the width and depth of sulci).

Most studies of the fractal properties of the cerebral cortex have focused on computing whole-brain measures (i.e. generating one number which summarizes the entire hemisphere). While quantification of whole-brain complexity is useful, it is well established that the brain is not uniformly affected by either aging or by progressive neurodegenerative diseases such as Alzheimer's disease [20]-[22]. Consequently, performing a local (or regional) analysis may allow for more specific and sensitive cortical complexity changes to be identified that represent the structural influence of non-uniformly distributed cerebral pathology. The purpose of this paper is to describe a technique for computing the local fractal dimension of the human cerebral cortex as extracted from high-resolution magnetic resonance imaging scans.

Having a measure of local fractal dimension could serve a number of very useful clinical purposes. First, the measure would provide a reliable quantification to the otherwise subjective and qualitative assessment of cortical shape. Currently used terms for assessing the integrity of the cerebral cortex, such as "mild age-appropriate atrophy", can be augmented with a quantitative, statistically based measure. Furthermore, the local fractal dimension measure is ideal for identifying subtle changes in brain shape that could be difficult to identify visually. Finally, subtle changes in brain complexity may herald the onset of neurological disease. As such, using the fractal analysis technique to identify structural changes in cortical complexity has potential for clinical use as a neuroimaging biomarker.

\section{Methods}

\subsection{Source Data}

The raw data used in this study were extracted from high-resolution, high-contrast magnetic resonance images (MP-RAGE sequence, resolution of $1 \times 1 \times 1.25 \mathrm{~mm}, \mathrm{TR}=9.7 \mathrm{~ms}$, TE $=4 \mathrm{~ms}$, flip angle $=10$ degrees, $\mathrm{T} 1=20$ $\mathrm{ms}$, and TD $=200 \mathrm{~ms}$ ). The images were obtained from the clinical database of magnetic resonance images at the University of Utah. All images were de-identified according to Institutional Review Board protocol. For the analysis of region size, 10 age-matched healthy adult subjects were selected. For demonstration of the potential clinical application, one healthy adult subject and one subject with clinically diagnosed Alzheimer's disease were selected.

\subsection{Image Pre-Processing}

In brief, we generate three-dimensional models of the cerebral cortex from high-contrast magnetic resonance images using a well-accepted image segmentation program called FreeSurfer. The methods for model generation have been well documented in other publications [23]-[27]. After the original images undergo motion correction and intensity normalization, the extracerebral voxels (or 3D pixels) are removed using a "skull-stripping” procedure. The 3D surfaces are generated based on the intensity values and geometric structure of the cortex (Figure 1(A)). The resulting surface has sub-millimeter accuracy [23] [25]. Each 3D surface is composed of tessellated triangles ( 200,000 per hemisphere). 


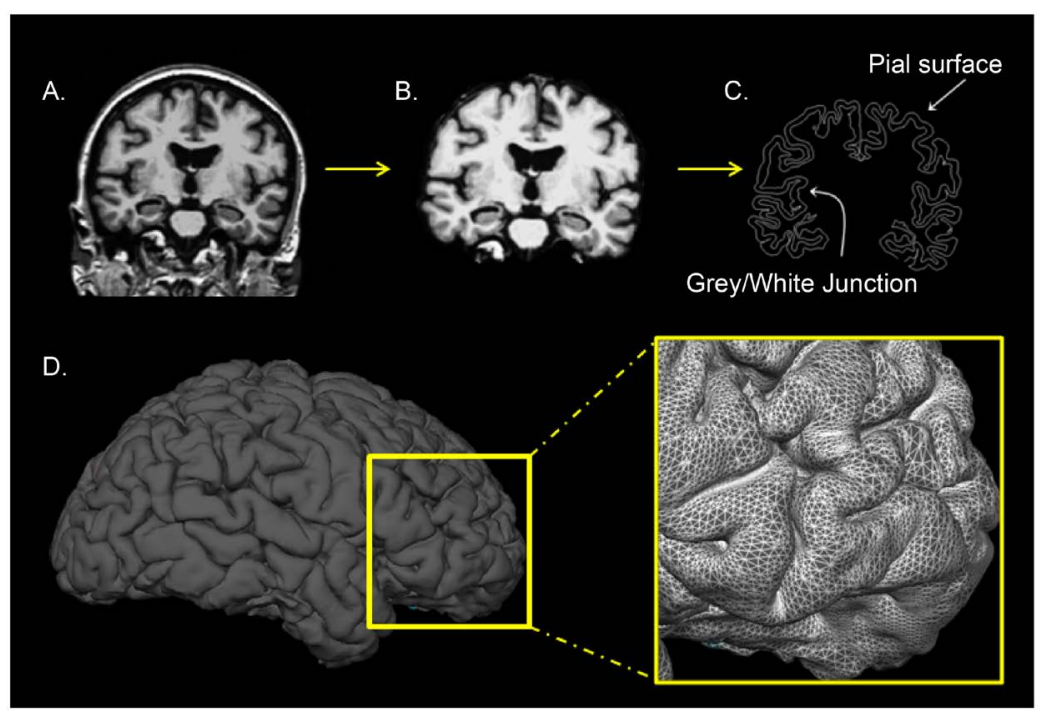

Figure 1. Image pre-processing. 3D models of the cortical surface were generated from high-contrast magnetic resonance images (MP-RAGE sequence) using the FreeSurfer pipeline. A. Original image (in coronal orientation); B. Skull-stripped image with neck removed and intensities normalized; C. 2D projection of the pial and grey/white surface; D. 3D view of the right hemisphere pial surface with a zoom-in on the frontal lobe to illustrate the triangular mesh.

\subsection{Computing Local Fractal Dimension Using Cube Counting}

The 3D Fractal Dimension $\left(f_{3 \mathrm{D}}\right)$ of the cortical surfaces is computed using a 3D cube-counting algorithm. This algorithm has been found to be a robust and accurate method of computing cortical complexity [6] [8] [14] [18] [19] [28]-[31]. This approach is derived from the Minkowski-Bouligand dimension with an extrapolation using $3 \mathrm{D}$ cubes instead of 2D boxes. Note that $f_{3 \mathrm{D}}$ is a unit-less measure. Initially, the 3D surface is tiled with cubes of a uniform size. The cube size is then changed, and the intersection computation is repeated. $f_{3 \mathrm{D}}$ is computed as the change in the log of the cube count divided by the change in the log of the cube size. See Equation (1).

$$
f_{3 D}=-\frac{\Delta \log (\text { cube count })}{\Delta \log (\text { cube size })}
$$

The range of cube sizes depends upon the resolution of the original data $(1 \mathrm{~mm}$ for the images used in this paper) and the upper size limit of the object to be measured. The human brain is around $150 \mathrm{~mm}$ long. The brain has self-similar fractal properties over a limited scale, which for this paper ranged $0.5 \mathrm{~mm}$ to $30 \mathrm{~mm}$ [19]. The best fit of any tiling could be established by repeating the tiling calculation multiple times with a slight random jitter of the relative position of the tiling cubes to the tessellated mesh.

Cube counting in this implementation requires the user to compute the intersection between a tiling grid of fractal counting cubes and the tessellated triangular mesh of the cortical surface. An algorithm for performing an intersection computation between one side of a counting cube and one side of an individual triangular mesh element is shown in Figure 2. This process would need to be repeated for each side of the mesh element and each side of the cube to determine successful intersection or not. Each intersecting box should be identified only once.

The entire algorithm for computing the local fractal dimension of a cortical surface model is shown in the flowchart in Figure 3. Note that the process in Figure 2 is contained in a single box in the bottom right corner of Figure 3. The resolution of the final image depends upon the number of center points used to compute the local fractal dimension. For the purpose of this project, it was reasonable to create an image at the same resolution as the original MR image. Every voxel that was labeled as belonging to the cerebral cortex during the image segmentation process described above was used as a center point for this analysis. This generates approximately 600,000 data points per hemisphere. 


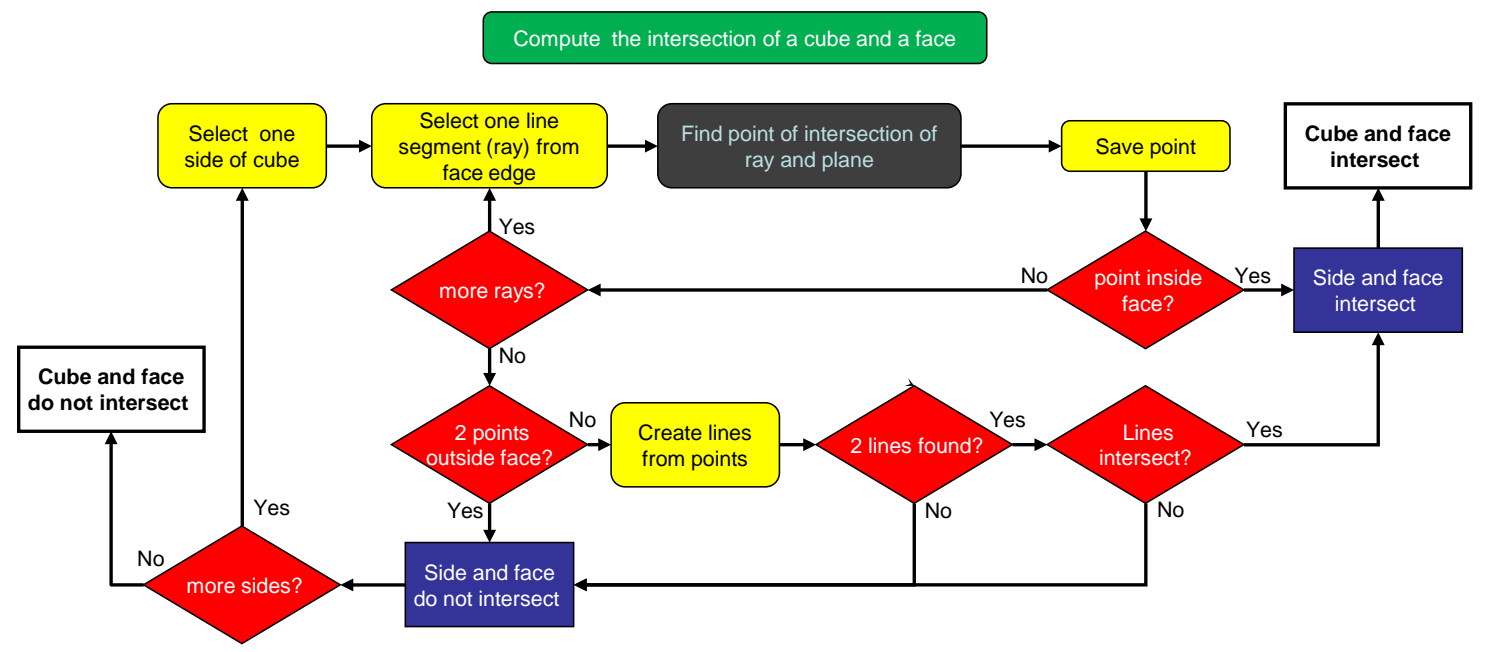

Figure 2. Flowchart for computing the intersection of side (edge of a triangular mesh element) with a face of a cube. The intersection is identified using standard geometry. Possible intersections for each triangular mesh side (3 edges) and each cube face (6 faces) are computed.
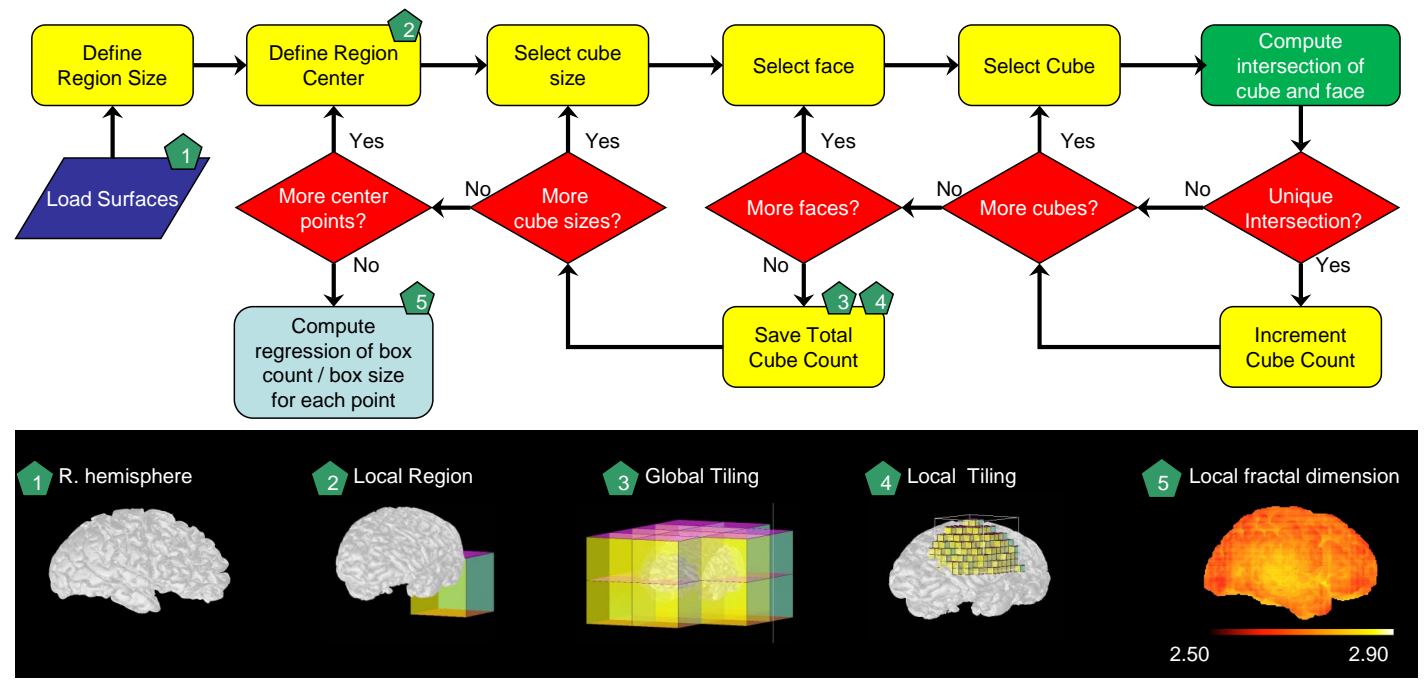

Figure 3. Flowchart for computing the local fractal dimension of the cerebral cortical surface. The flowchart for the cube-edge algorithm is shown in Figure 2. An example of a complete local fractal dimension computation is shown on the bottom left with local fractal values color-coded using the "heat" color scale.

\subsection{Determining the Effect of Region Size}

The size of the region to be analyzed will likely have a significant effect on the calculated regional variability. If the region size is very large ( $>0.5$ the size of the whole brain), then the regional variation will be averaged across a large swath of cortex, and local values will converge to global values. If the region size is very small $(<2 \times$ the image resolution), then there is insufficient data to compute the cube count to cube size ratio, and the resulting values will be unstable and non-anatomical (i.e. complexity values may be $>3.0$ or $<1.0$ ). Cubic regions with side lengths of 15,30 , and 60 mm were selected for this study.

\section{Results}

\subsection{Effects of Selecting a Region Size}

The local fractal dimension values for a representative individual brain using 3 different cubic region sizes (15, 30, and $60 \mathrm{~mm}$ side lengths) are shown in Figure 4. All 10 images produced similar results. When the smaller 


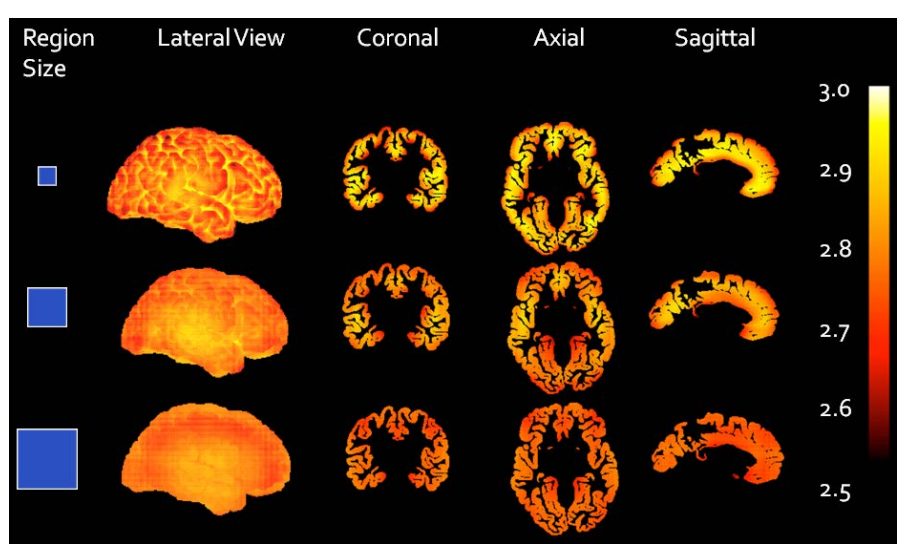

Figure 4. Local fractal values of a cortical surface as a function of region size. A tessellated mesh was tiled with $1 \mathrm{~mm}$ cubes, each of which was used as a center point for computing the local fractal dimension. The region size side length varied from $15 \mathrm{~mm}$ to $60 \mathrm{~mm}$ (right column blue boxes). A lateral view of the right hemisphere is shown in the second column tiled with cubes colored according to the computed local fractal dimension (heat color scale). The scale bar for the local fractal dimension (unit-less) is shown on the right. To visualize the internal structure, slices of the cortex are shown for a mid-thalamic coronal view, an axial view through the genu of the internal capsule, and a sagittal slice $2 \mathrm{~mm}$ to the right of midline. The 30 $\mathrm{mm}$ region size demonstrates the greatest variability in local fractal dimension.

region size $(15 \mathrm{~mm})$ is selected, the local fractal values largely reflect cortical density within the region. Using the heat color scale, regions centered close to the inner or outer edges appear cooler, while regions in the center of the cortex are warmer. When measuring at this relatively small scale, there is not much regional variability across the cortex. When using the larger region size $(60 \mathrm{~mm})$, the local regions again show relatively little variation as the fractal values converges towards the global value (2.72 in this case). The intermediate region size (30 $\mathrm{mm}$ ) shows the most local variation of the three region sizes.

\subsection{Clinical Application}

To demonstrate the ability of this local fractal dimension tool to identify regional variation in brain structure, cortical fractal dimension values were calculated for two adult subjects (30 $\mathrm{mm}$ region size). The results are shown in Figure 5. One of these subjects has been diagnosed clinically with a progressive neurodegenerative condition (Alzheimer's disease, which is rated as mild in severity) and has known structural deficits identified on his magnetic resonance images. A healthy adult subject is shown for comparison. For the healthy adult subject, there is some regional variability again noted, but no local complexity values fall below 2.5. For the subject with Alzheimer's disease, there is a generalized loss of complexity noted (color scale shifted towards the red direction on the heat scale) and there are prominent areas of marked decreased complexity in the bilateral parietal lobes and medial temporal lobes.

\section{Discussion}

This paper has demonstrated a method for computing the local fractal dimension of the human cerebral cortex as extracted from high resolution magnetic resonance images. The value of local fractal dimension depends upon the region size that is used. An intermediate region size (30 $\mathrm{mm}$ in this example) yields results that capture the variation in local complexity. Using an intermediate region size, the local variation in cortical fractal dimension reflects clinically significant structural factors. For example, cerebral atrophy associated with neurodegenerative conditions, such as Alzheimer's disease, causes decreases in the calculated value of the local fractal dimension. Moreover, the heterogeneous spatial patterns of cerebral atrophy associated with a neurodegenerative disease are also reflected in the pattern of local fractal dimension change. This proof of concept demonstrates potential use 


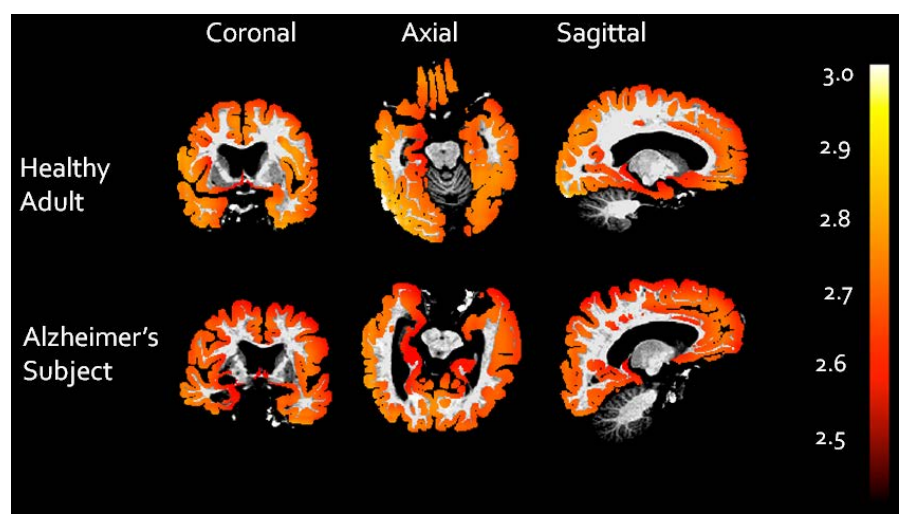

Figure 5. Local fractal values of the cortex decrease with Alzheimer's disease. Shown are coronal, axial, and sagittal images of the brain with the local fractal dimension of the cortex superimposed. The scale bar for the local fractal dimension (unit-less) is shown on the right. The first row comes from a healthy adult subject, and the second row is a subject with a clinical diagnosis of Alzheimer's disease. Both subjects are 75 years old. The fractal dimension values of the Alzheimer's subject are lower than the healthy adult, with a predominance noted in the medial temporal lobes and bilateral parietal lobes.

of local fractal dimension as a biomarker for changes in cortical structure.

Statistical analysis of this local fractal data will require implementation of an alignment algorithm in order to compare analogous regions of multiple brains. Using affine alignment techniques is unlikely to result in an accurate image (e.g. the local fractal surfaces are too irregular compared to each other). One method would be to use a spherical mapping approach, such as the one implemented by FreeSurfer.

In addition, the values for local fractal dimension need to be normalized against a background of normal aging to determine the normal range of local values for any given age group. This critical calibration then allows the data for an individual to be compared to an age-matched population to determine the statistical significance of the local variability. This step will also be critical to determine which local variations are common in the population (and thus without clinical significance) and which variations are associated with neuropathology. Ideally such changes could be identified early in the course of the disease and could identify those at risk for future symptomatic progression.

Local fractal dimension is one of many measures which can be used to quantify the integrity of the cerebral cortex. The technique has the advantage of quantifying measures of cortical shape, which is complementary to volumetric measures. With further development and testing, this tool may allow new and clinically useful assessments of neuroimaging data to help physicians better diagnose and manage neurodegenerative disease.

\section{Acknowledgements}

This work was generously supported by grants from the Robert Wood Johnson Foundation, the American Federation for Aging Research, the Hartford Foundation, and the National Institute on Aging (K23-AG038356). Additional support has come from the Center for Alzheimer's Care, Imaging and Research and the Department of Neurology at the University of Utah. The author would like to thank Mr. Sourav Kole for his assistance in proofreading this paper. Additional gratitude is extended to collaborators and members of my mentoring team, including Drs. Norman Foster, Mark Supiano, Sarang Joshi, P. Thomas Fletcher, Denise Park, Kristen Kennedy, Karen Rodrigue, and Angela Wang. The author would also like to acknowledge technical assistance in software development from Mr. Benjamin Tyler and Mr. Michael Hwang.

\section{References}

[1] Mandelbrot, B.B. (1982) The Fractal Geometry of Nature. W.H. Freeman, San Francisco.

[2] Mandelbrot, B.B. (1977) Fractals: Form, Chance, and Dimension. W. H. Freeman, San Francisco. 
[3] Bullmore, E., Brammer, M., Harvey, I., et al. (1994) Fractal Analysis of the Boundary between White Matter and Cerebral Cortex in Magnetic Resonance Images: A Controlled Study of Schizophrenic and Manic-Depressive Patients. Psychological Medicine, 24, 771-781. http://dx.doi.org/10.1017/S0033291700027926

[4] Free, S.L., Sisodiya, S.M., Cook, M.J., Fish, D.R. and Shorvon, S.D. (1996) Three-Dimensional Fractal Analysis of the White Matter Surface from Magnetic Resonance Images of the Human Brain. Cereb Cortex, 6, 830-836. http://dx.doi.org/10.1093/cercor/6.6.830

[5] Hofman, M.A. (1991) The Fractal Geometry of Convoluted Brains. Journal für Hirnforschung, 32, $103-111$.

[6] Im, K., Lee, J.M., Yoon, U., et al. (2006) Fractal Dimension in Human Cortical Surface: Multiple Regression Analysis with Cortical Thickness, Sulcal Depth, and Folding Area. Human Brain Mapping, 27, 994-1003. http://dx.doi.org/10.1002/hbm.20238

[7] Kiselev, V.G., Hahn, K.R. and Auer, D.P. (2003) Is the Brain Cortex a Fractal? Neuroimage, 20, 1765-1774. http://dx.doi.org/10.1016/S1053-8119(03)00380-X

[8] Majumdar, S. and Prasad, R.R. (1988) The Fractal Dimension of Cerebral Surfaces Using Magnetic Resonance Imaging. Computers in Physics, 2, 69-73. http://dx.doi.org/10.1063/1.168314

[9] Ha, T.H., Yoon, U., Lee, K.J., et al. (2005) Fractal Dimension of Cerebral Cortical Surface in Schizophrenia and Obsessive-Compulsive Disorder. Neuroscience Letters, 384, 172-176. http://dx.doi.org/10.1016/j.neulet.2005.04.078

[10] Sandu, A.L., Rasmussen Jr., I.A., Lundervold, A., et al. (2008) Fractal Dimension Analysis of MR Images Reveals Grey Matter Structure Irregularities in Schizophrenia. Computerized Medical Imaging and Graphics, 32, 150-158.

[11] Casanova, M.F., Daniel, D.G., Goldberg, T.E., Suddath, R.L. and Weinberger, D.R. (1989) Shape Analysis of the Middle Cranial Fossa of Schizophrenic Patients. A Computerized Tomographic Study. Schizophrenia Research, 2, 333-338. http://dx.doi.org/10.1016/0920-9964(89)90024-8

[12] Casanova, M.F., Goldberg, T.E., Suddath, R.L., et al. (1990) Quantitative Shape Analysis of the Temporal and Prefrontal Lobes of Schizophrenic Patients: A Magnetic Resonance Image Study. The Journal of Neuropsychiatry and Clinical Neurosciences, 2, 363-372.

[13] Wu, Y.T., Shyu, K.K., Jao, C.W., et al. (2010) Fractal Dimension Analysis for Quantifying Cerebellar Morphological Change of Multiple System Atrophy of the Cerebellar Type (MSA-C). Neuroimage, 49, 539-551.

[14] Cook, M.J., Free, S.L., Manford, M.R., et al. (1995) Fractal Description of Cerebral Cortical Patterns in Frontal Lobe Epilepsy. European Neurology, 35, 327-335. http://dx.doi.org/10.1159/000117155

[15] Pirici, D., Mogoanta, L., Margaritescu, O., et al. (2009) Fractal Analysis of Astrocytes in Stroke and Dementia. Romanian Journal of Morphology and Embryology, 50, 381-390.

[16] Thompson, P.M., Lee, A.D., Dutton, R.A., Geaga, J.A., Hayashi, K.M., Eckert, M.A., et al. (2005) Abnormal Cortical Complexity and Thickness Profiles Mapped in Williams Syndrome. The Journal of Neuroscience, 25, 4146-4158. http://dx.doi.org/10.1523/JNEUROSCI.0165-05.2005

[17] Wu, Y.T., Shyu, K.K., Chen, T.R. and Guo, W.Y. (2009) Using Three-Dimensional Fractal Dimension to Analyze the Complexity of Fetal Cortical Surface from Magnetic Resonance Images. Nonlinear Dynamics, 58, 745-752. http://dx.doi.org/10.1007/s11071-009-9515-y

[18] King, R.D., George, A.T., Jeon, T., Hynan, L.S., Youn, T.S., Kennedy, D.N., et al. (2009) Characterization of Atrophic Changes in the Cerebral Cortex Using Fractal Dimensional Analysis. Brain Imaging and Behavior, 3, 154-166. http://dx.doi.org/10.1007/s11682-008-9057-9.

[19] King, R.D., Brown, B., Hwang, M., Jeon, T. and George, A.T. (2010) Fractal Dimension Analysis of the Cortical Ribbon in Mild Alzheimer's Disease. Neuroimage, 53, 471-479.

[20] Walhovd, K.B., Westlye, L.T., Amlien, I., Espeseth, T., Reinvang, I., Raz, N., et al. (2011) Consistent Neuroanatomical Age-Related Volume Differences across Multiple Samples. Neurobiology of Aging, 32, 916-932.

[21] Raz, N., Lindenberger, U., Rodrigue, K.M., Kennedy, K.M., Head, D., Williamson, A., et al. (2005) Regional Brain Changes in Aging Healthy Adults: General Trends, Individual Differences and Modifiers. Cereb Cortex, 15, 16761689.

[22] Raz, N., Ghisletta, P., Rodrigue, K.M., Kennedy, K.M. and Lindenberger, U. (2010) Trajectories of Brain Aging in Middle-Aged and Older Adults: Regional and Individual Differences. Neuroimage, 51, 501-511.

[23] Dale, A.M., Fischl, B. and Sereno, M.I. (1999) Cortical Surface-Based Analysis. I. Segmentation and Surface Reconstruction. Neuroimage, 9, 179-194. http://dx.doi.org/10.1006/nimg.1998.0395

[24] Fischl, B., Sereno, M.I. and Dale, A.M. (1999) Cortical Surface-Based Analysis. II: Inflation, Flattening, and a Surface-Based Coordinate System. Neuroimage, 9, 195-207. http://dx.doi.org/10.1006/nimg.1998.0396

[25] Fischl, B. and Dale, A.M. (2000) Measuring the Thickness of the Human Cerebral Cortex from Magnetic Resonance Images. Proceedings of the National Academy of Sciences of the United States of America, 97, 11050-11055. 
http://dx.doi.org/10.1073/pnas.200033797

[26] Fischl, B., van der Kouwe, A., Destrieux, C., Halgren, E., Ségonne, F., Salat, D.H., et al. (2004) Automatically Parcellating the Human Cerebral Cortex. Cereb Cortex, 14, 11-22. http://dx.doi.org/10.1093/cercor/bhg087

[27] Han, X., Jovicich, J., Salat, D., van der Kouwe, A., Quinn, B., Czanner, S., et al. (2006) Reliability of MRI-Derived Measurements of Human Cerebral Cortical Thickness: The Effects of Field Strength, Scanner Upgrade and Manufacturer. Neuroimage, 32, 180-194.

[28] Esteban, F.J., Sepulcre, J., de Mendizabal, N.V., Goñi, J., Navas, J., de Miras, J.R., et al. (2007) Fractal Dimension and White Matter Changes in Multiple Sclerosis. Neuroimage, 36, 543-549.

[29] Lee, J.M., Yoon, U., Kim, J.J., Kim, I.Y., Lee, D.S., Kwon, J.S., et al. (2004) Analysis of the Hemispheric Asymmetry Using Fractal Dimension of a Skeletonized Cerebral Surface. IEEE Transactions on Biomedical Engineering, 51, 1494- 1498. http://dx.doi.org/10.1109/TBME.2004.831543

[30] Zhang, L., Dean, D., Liu, J.Z., Sahgal, V., Wang, X. and Yue, G.H. (2007) Quantifying Degeneration of White Matter in Normal Aging Using Fractal Dimension. Neurobiology of Aging, 28, 1543-1555.

[31] Zhang, L., Liu, J.Z., Dean, D., Sahgal, V. and Yue, G.H. (2006) A Three-Dimensional Fractal Analysis Method for Quantifying White Matter Structure in Human Brain. Journal of Neuroscience Methods, 150, 242-253. http://dx.doi.org/10.1016/j.jneumeth.2005.06.021 
Scientific Research Publishing (SCIRP) is one of the largest Open Access journal publishers. It is currently publishing more than 200 open access, online, peer-reviewed journals covering a wide range of academic disciplines. SCIRP serves the worldwide academic communities and contributes to the progress and application of science with its publication.

Other selected journals from SCIRP are listed as below. Submit your manuscript to us via either submit@scirp.org or Online Submission Portal.
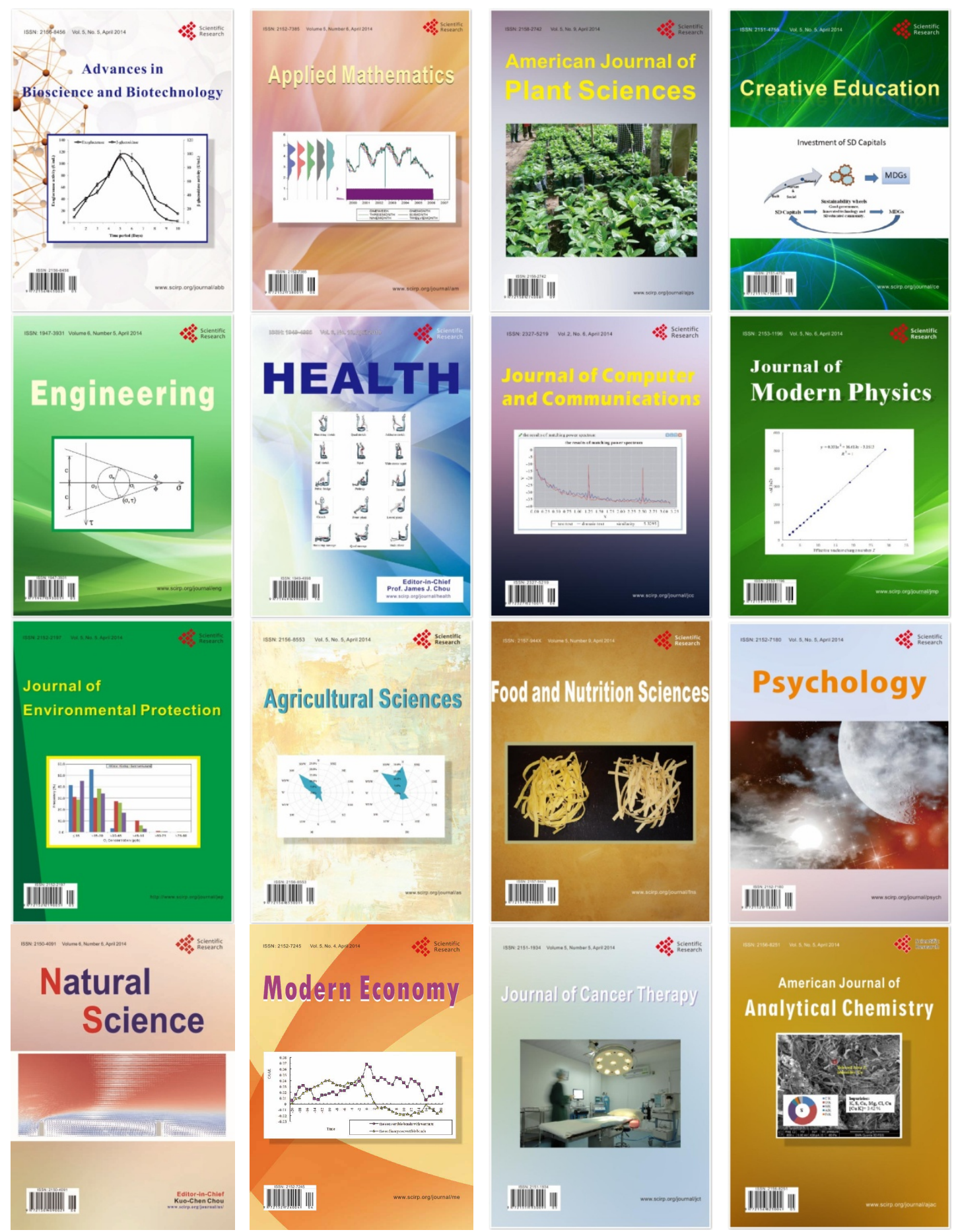